\title{
Metodología de muestreo de suelos con fines de análisis químico para la detección de metales pesados
}

\section{Soil sampling methodology for chemical analysis purposes for the detection of heavy metals}

\author{
Liz Jhoana Astonitas Carrasco ${ }^{1 *}\left(\mathbb{D}\right.$, Elí Pariente Mondragón ${ }^{1,2}$ (D), Manuel Emilio Milla Pino ${ }^{3}$ (D)
}

\section{RESUMEN}

Una metodología adecuada de muestreo es crucial para determinar si un sitio está contaminado o no, y si la magnitud de la contaminación requiere una intervención para proteger la salud humana y la integridad de los ecosistemas. Por lo tanto, las muestras recolectadas para los análisis deben ser relevantes y verdaderamente representativas, siendo el aspecto más crítico de un programa de muestreo. El presente trabajo tiene como objetivo establecer una metodología de muestreo de suelos agrícolas para el análisis de metales. Esta propuesta metodológica de muestreo consta de cinco etapas: Muestreo preliminar del área de estudio, cuya finalidad es detectar homogeneidad o heterogeneidad en sus características fisicoquímicas del suelo; muestreo definitivo del área de estudio, el mismo que consta de una segmentación, estratificación del área de estudio y distribución de puntos de muestreo; Plan de muestreo; recolección de muestras y análisis de las muestras.

Palabras clave: métodos, plan de muestreo, suelos agrícolas, metales, análisis químico.

\begin{abstract}
Proper sampling methodology is crucial to determine whether a site is contaminated or not, and whether the magnitude of the contamination requires intervention to protect human health and the integrity of ecosystems. Therefore, the samples collected for analysis must be relevant and truly representative, being the most critical aspect of a sampling program. The objective of this work is to establish a methodology for sampling agricultural soils for the analysis of metals. This methodological sampling proposal consists of five stages: Preliminary sampling of the study area, whose purpose is to detect homogeneity or heterogeneity in its physicochemical characteristics of the soil; definitive sampling of the study area, which consists of a segmentation, stratification of the study area and distribution of sampling points; Sampling plan; collection of samples and analysis of samples.
\end{abstract}

Keywords: methods, sampling plan, agricultural soils, metals, chemical análisis.

\footnotetext{
${ }^{1}$ Universidad Nacional Toribio Rodríguez de Mendoza de Amazonas, Facultad de Ingeniería y Ciencias Agrarias, Laboratorio de Dendrología y Herbario, Chachapoyas, Perú.

${ }^{2}$ Universidad Nacional Toribio Rodríguez de Mendoza de Amazonas, Instituto de Investigación para el Desarrollo Sustentable de Ceja de Selva, Chachapoyas, Perú.

${ }^{3}$ Universidad Nacional de Jaén, Facultad de Ingeniería Civil, Jaén, Perú.

"Autor de correspondencia.E-mail:jhoanaweb@hotmail.com
} 


\section{INTRODUCCIÓN}

El suelo es un recurso natural renovable agotable, que cumple una variedad de funciones vitales en nuestra sociedad (Kelepertzis, 2014); entre las principales, almacenamiento y filtración de agua, sostenimiento de la biodiversidad, ciclo de nutrientes, regulación del clima y producción de alimentos (Burbano, 2016). Este recurso es la base para la agricultura y el medio en el que se desarrollan casi todas las plantas de las que obtenemos alimentos (FAO, 2015). Sin embargo, en los últimos años las malas prácticas agrícolas han provocado el uso excesivo de productos agroquímicos (fertilizantes y plaguicidas) (Gómez, 2008; Sharafati et al., 2016), las mismas que pueden causar degradación de los suelos, destruir poblaciones de microorganismos que actúan como controladores biológicos y contaminar cuerpos de agua (Rueda et al., 2011). Además, Kelepertzis (2014) muestra que la aplicación excesiva de productos agroquímicos contribuye significativamente en la elevación de concentraciones de metales pesados en suelos agrícolas.

Los metales pesados constituyen un serio peligro para la humanidad, una vez en el suelo, siguen varias vías que conducen a las cadenas tróficas (Delince et al., 2015). Muchos de los metales (As, Cd, Pb) pueden llegar hasta el ser humano a través del consumo de alimentos atentando contra su salud (Peralta et al., 2009). El interés por los metales pesados en los suelos agrícolas está relacionado con su capacidad de acumulación en el perfil del suelo hasta concentraciones tóxicas, y riesgos para la salud humana, no obstante, su biodegradabilidad y su interacción con las diferentes propiedades del suelo que determinan su acumulación, movilidad y biodisponibilidad hacia otros componentes del ecosistema (Rueda et al., 2011).

Ante la necesidad de manejar y conservar el suelo, la comunidad científica internacional y las autoridades a nivel mundial han planteado establecer indicadores de calidad del mismo para conservarlo y mejorar la productividad de manera sostenible (Guzmán et al., 2019). En el Perú el Ministerio del Ambiente aprobó los Estándares de Calidad Ambiental (ECA) para Sue- los mediante Decreto Supremo N 011-2017-MINAM, donde se establecen parámetros de calidad ambiental en suelos, estos valores se sustentan en un análisis de factores que inciden en la salud pública y calidad ambiental, y se basan en las últimas investigaciones científicas, así como en los estándares que establecen organizaciones internacionales (MINAM, 2017).

La aplicación de los ECAs exige la realización de análisis de suelo, cuyos resultados deben ser comparados con los parámetros establecidos en la normativa para la toma de decisiones; estos análisis nos permiten determinar el estado de fertilidad del suelo (Pérez, 2013). Sin embargo, a nivel nacional no existe una metodología o un procedimiento homogéneo de muestreo de suelos agrícolas para determinar el contenido de metales. Una metodología adecuada de muestreo es crucial para determinar si un sitio está contaminado o no, y si la magnitud de la contaminación requiere una intervención para proteger la salud humana y la integridad de los ecosistemas; es decir, la estrategia del muestreo, desempeñan un papel sumamente importante para la adecuada evaluación del sitio y su respectiva gestión (MINAM, 2014). Por lo tanto, las muestras recolectadas para los análisis deben ser relevantes y verdaderamente representativas, siendo el aspecto más crítico de un programa de muestreo (Diaz et al., 2005).

Este artículo tiene por objetivo describir una metodología de muestreo que puede ser utilizada en los procesos de muestreo y análisis químico de muestras para determinar el contenido de metales en suelos agrícolas.

\section{METODOLOGÍA PROPUESTA}

La propuesta de muestreo de suelos para análisis de metales tiene por finalidad disponer de una base metodológica que permita a los investigadores obtener información confiable y de calidad de los sitios estudiados.

En la Figura 1 se muestran las etapas de la propuesta metodológica de muestreo de suelos agrícolas.

\section{Etapa 1: Muestreo preliminar del área de estudio}

Previo a la obtención de muestras para el análisis de metales en los suelos agrícolas, es necesario realizar un muestreo preliminar donde se analicen las caracte- 


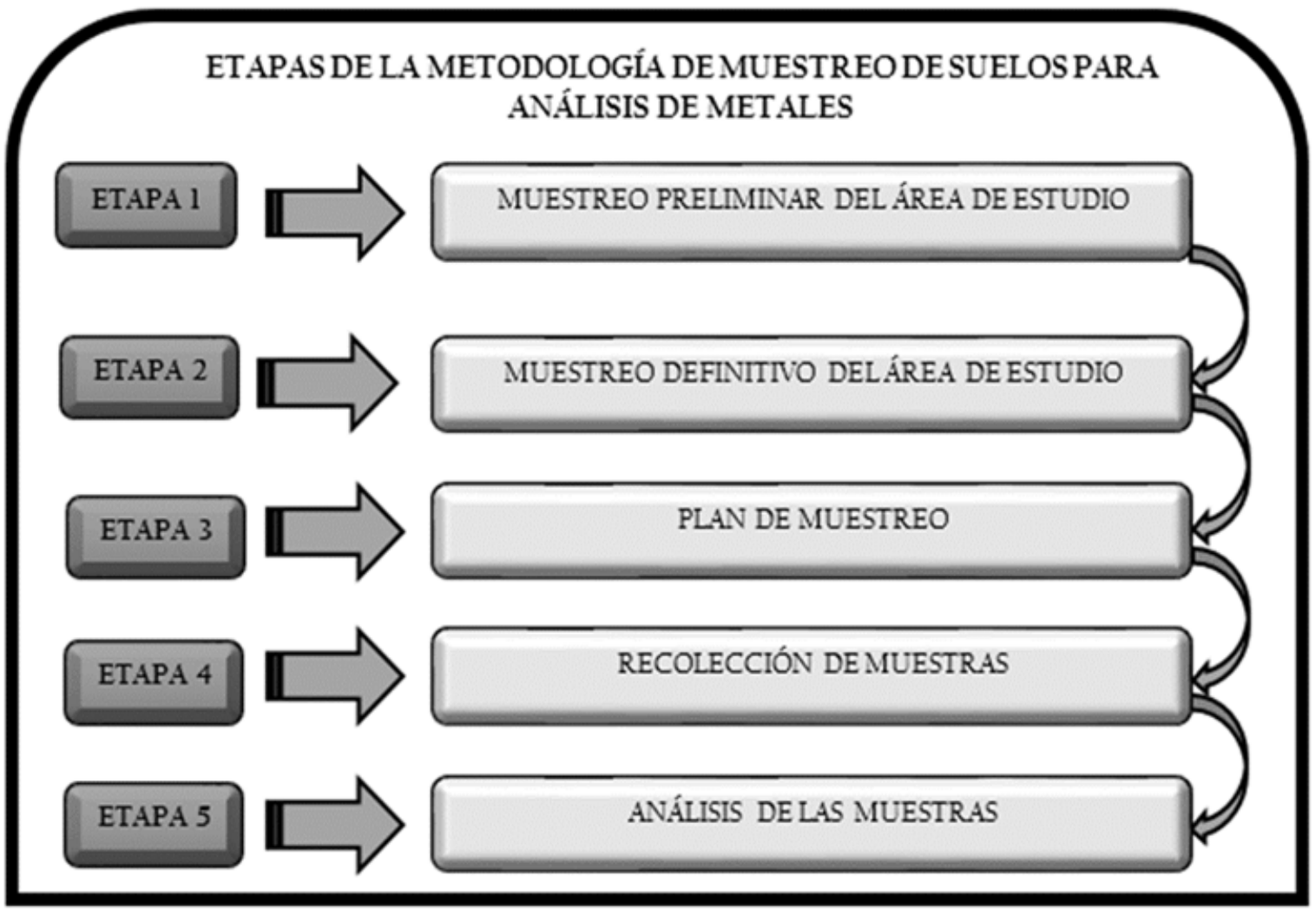

Figura 1. Etapas de la metodología de muestreo de suelos.

rísticas fisicoquímicas (pH, Conductividad Eléctrica, Materia Orgánica, Capacidad de Intercambio Catiónico y el Análisis Mecánico) del área de estudio.

El muestreo preliminar del área de estudio está basado en detectar homogeneidad o heterogeneidad en los parámetros físicos y químicos de los suelos. Este muestreo determinará el grado de asociación y diferencias en las características del suelo; con la finalidad de detectar características de similitudes o diferencias determinantes para un muestreo definitivo.

Para la selección de los sitios de muestreo, se establecen condiciones que se deberían cumplir para lograr con los objetivos de cada estudio realizado. Las condiciones que se establecen son las siguientes:

- Dentro del área de estudio, establecer dos parcelas de suelos agrícolas de 1 hectárea cada una, las mismas que deben estar ubicadas a una distancia considerable dentro del sitio de estudio puesto que la finalidad del muestreo preliminar es detectar homogeneidad o heterogeneidad en las características fisicoquímicas del suelo estudiado.
- Tomar muestras de suelos de los extremos y parte central de cada parcela seleccionada (Figura. 2).

- Georreferenciar los puntos muestreados.

- Analizar en laboratorio cada muestra de suelo recolectado de manera individual para determinar sus parámetros físicos y químicos.

- Las muestras deben ser colectadas antes de la siembra o después de la cosecha de los cultivos, con la finalidad de evitar sesgo en los datos físicos y químicos por fertilizantes o manejo agroquímico del área. 


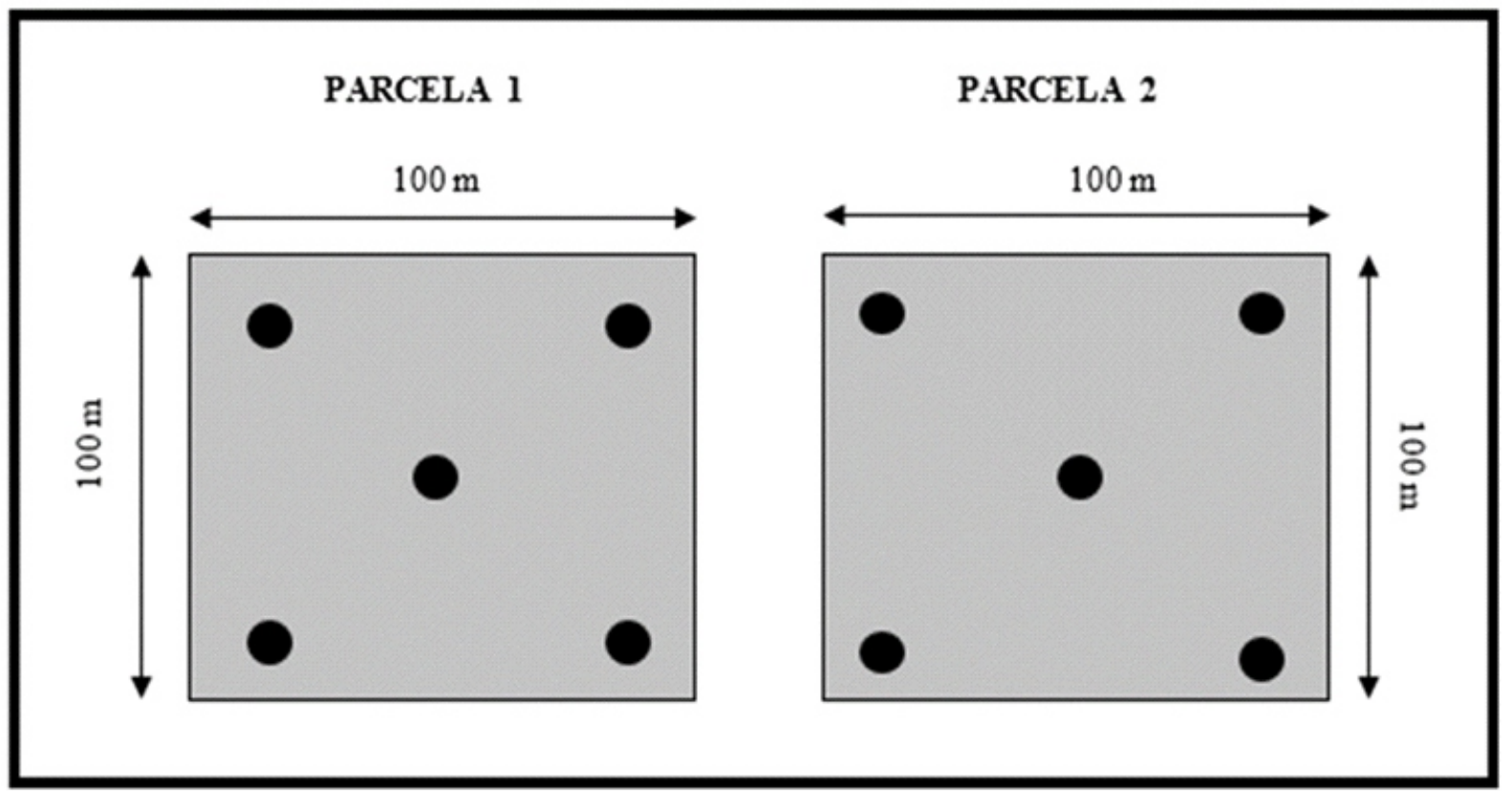

Figura 2. Distribución de los puntos de muestreo en cada parcela.

\section{Etapa 2: Muestreo definitivo del área de estudio}

Previo a realizar el muestreo definitivo se debe realizar una segmentación del área de estudio, específicamente de las áreas con cultivos agrícolas a estudiar.

Realizar una estratificación del área de estudio con la finalidad de garantizar que todos los segmentos que conforman la población estén debidamente representados en la muestra. El área de estudio se puede estratificar de acuerdo a rangos altitudinales, los mismos que va a depender del sitio estudiado y de las altitudes en las que se desarrolla un determinado cultivo. Es recomendable estratificar un área como mínimo en tres zonas (zona alta, media y baja). La estratificación se puede realizar con la ayuda del software ArcGIS, utilizando un Modelo de Elevación Digital (DEM) ALOS PALSAR de 12.5 metros de resolución, y finalmente realizar una reclasificación de acuerdo a los rangos altitudinales establecidos de acuerdo al objetivo de la investigación.

La distribución de puntos de muestreo para determinar la concentración de metales en los suelos agrícolas se debe realizar teniendo en cuenta dos aspectos importantes:

La zona con mayor superficie de parcelas agrícolas sea la que aporte con la mayor cantidad de muestras al estudio.

Los resultados del análisis preliminar (caracterización de suelos): los resultados del análisis fisicoquímico son determinantes en esta etapa, ya que, si los parámetros fisicoquímicos del suelo de ambas parcelas analizadas muestran características heterogéneas, entonces el muestreo definitivo comprendería al área de estudio en su totalidad, lo que indica una mayor cantidad de muestras; por el contrario si los resultados indican características fisicoquímicas homogéneas, en el muestreo definitivo las muestras a recolectar sería menor.

Un caso concreto donde es posible utilizar esta propuesta metodológica de muestreo es cuando el objetivo de la investigación es determinar el contenido de metales en suelos agrícolas, cuya área de estudio está situado a ambos márgenes de un río. En este caso, las parcelas para el muestreo preliminar donde se analizan las características fisicoquímicas serían seleccionadas en ambos márgenes del río (una parcela de una hectárea en cada margen). Finalmente, los resultados del muestreo preliminar determinarían el muestreo definitivo ya que, si las características fisicoquímicas del suelo de ambos márgenes son heterogéneas, el muestreo definitivo se realizaría en ambos márgenes del río. Por el contrario, si los resultados indican características homogéneas, el muestreo para evaluar los contaminantes en los suelos se realizaría únicamente en un margen del río, ya sea el margen derecho o el margen izquierdo. 


\section{Datos generales}

- Objetivo del muestreo: definir de manera clara y precisa las metas que se desean cumplir.

- Localización del sitio: indicar el país, departamento, provincia, distrito, centro poblado. Incluir planos o imágenes satelitales. Indicar coordenadas UTM.

- Delimitación de la zona de muestreo: coordenadas UTM debidamente verificados con planos oficiales y/o acercamiento de imágenes satelitales, trazo del polígono a estudiar.

- Parámetros a estudiar: (metales)

- Norma de referencia para el muestreo: guía para el muestreo de suelos, MINAM.

\section{Planeación y procedimiento del muestro}

- $\quad$ Tipo de muestreo: muestreo de identificación (depende del objetivo de estudio).

- Localización, distribución y número de muestras: se empleará un muestreo estadístico aleatorio estratificado y el número de muestras dependerá del área de estudio.

- Tipo de muestras: para el análisis de metales en suelos agrícolas es recomendable analizar muestras individuales.

- Estimación del número total de muestras: hace referencia al número de muestras, el mismo que está en función al área de estudio.

- Profundidad de muestreo: para análisis de metales en suelos agrícolas se recomienda a una profundidad de 0 a $30 \mathrm{~cm}$.

- Equipos de muestreo: hace referencia los equipos que se utilizarán en campo, tales como: pala, bolsa plástica, balanza, bolsas ziploc, GPS, entre otros.

- Equipos de protección personal de los involucrados en el muestreo: guantes, botas, entre otros.

- Tipo de recipiente y cantidad de la muestra: indicar el material del recipiente donde se recolectarán las muestras y la cantidad (0,5 kilogramos aproximadamente en cada muestra).

- Clave única de identificación de las muestras: a cada muestra se le debe asignar una clave o código para su fácil identificación.

- Laboratorio: indicar el laboratorio donde se realizará el análisis de las muestras recolectadas, el mismo que deberá estar debidamente acreditado.

\section{Etapa 3: Plan de muestreo}

Previo a la extracción de muestras, se debe elaborar un plan de muestreo. El Plan de muestreo es un documento formal que sirve para programar en forma lógica todas las actividades que están implícitas en un muestreo (MINAM, 2014). A continuación, se describe la estructura de un Plan de muestreo en la Tabla 1

\section{Etapa 4: Recolección de muestras de suelo}

Las muestras de suelo colectadas serán simples para cada punto propuesto. En cada punto de muestreo se registrarán las coordenadas geográficas mediante el uso de un GPS (Sistema de Posicionamiento Global).

La recolección de las muestras de suelo en cada punto de muestreo consistirá en un primer momento en la 


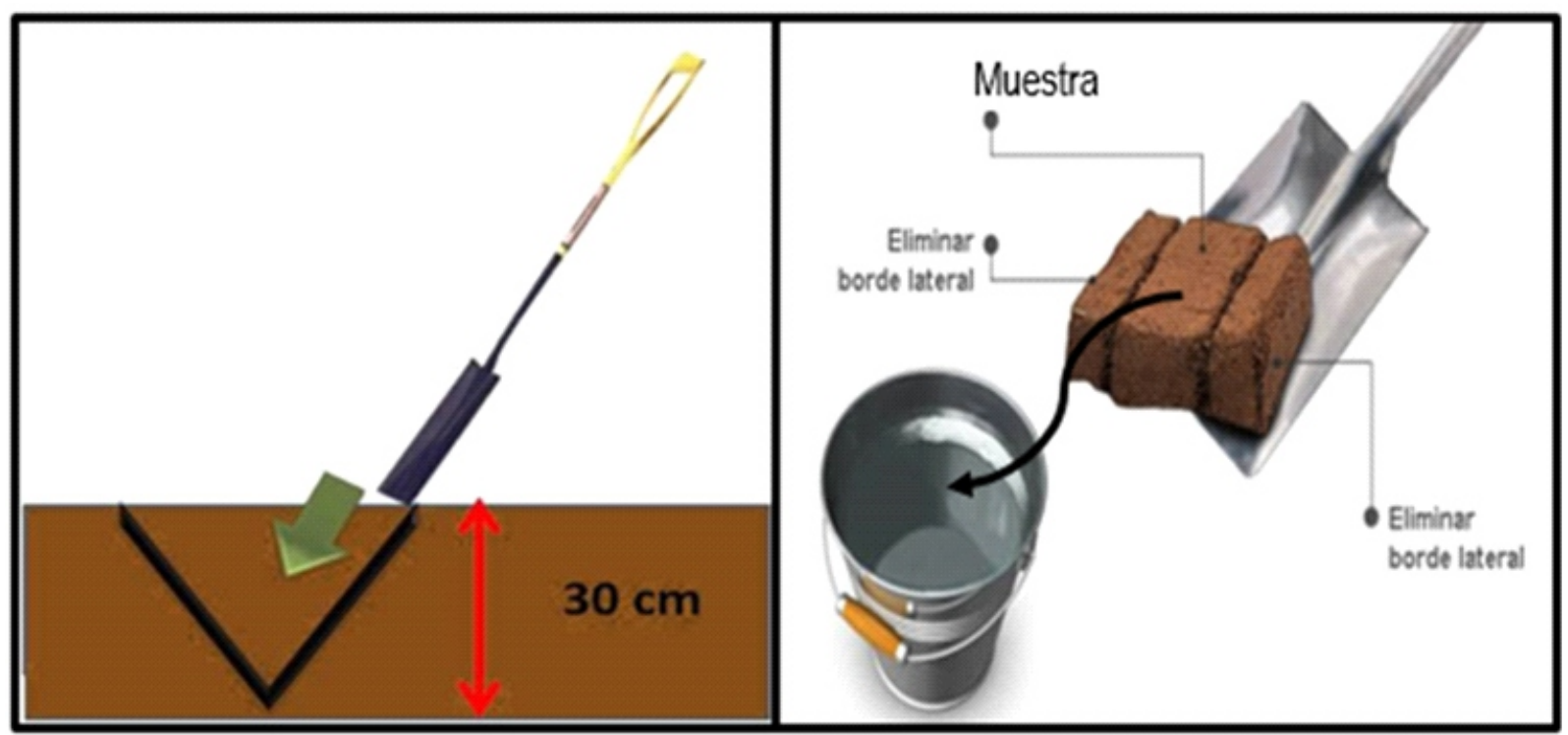

Figura 3. Procedimiento de recolección de muestras.

remoción de la cubierta vegetal mediante el uso de una pala, evitando extraer material del suelo. La profundidad de las muestras será de $0-30 \mathrm{~cm}$ por tratarse de suelos agrícolas, tal y como está establecido en la Guía para Muestreo de Suelos (MINAM, 2014); para ello, con la ayuda de una pala se realizarán cortes en el suelo en forma de "V", seguidamente se procederá a la eliminación de los bordes laterales del suelo extraído en la pala, quedando así únicamente la porción central de suelo lo que representa a la muestra (Figura 3).

Cada muestra estará conformada por 0,5 kilogramos de suelo, las cuales serán depositadas en bolsas ziploc debidamente rotuladas.

\section{Etapa 5: Análisis de las muestras}

Los análisis de metales en las muestras de suelos agrícolas deben ser realizados en un laboratorio debidamente acreditado.

\section{CONCLUSIONES}

En la actualidad no existe una metodología o procedimiento homogéneo de muestreo de suelos agrícolas para determinar el contenido de metales, siendo muchas veces una limitante para las investigaciones. La ausencia de una metodología de muestreo genera muchas veces la utilización de una gran diversidad de métodos no evaluados, con resultados poco confiables. Si bien es cierto, en los diversos países contamos con
Guías para Muestreos de Suelos. Sin embargo, en éstos se hace referencia de manera general a las técnicas de muestreo, manejo de las muestras y determinación de puntos de muestreo, pero no contamos con una metodología específica para el muestreo de suelos agrícolas para análisis de metales. La presente metodología de muestreo de suelos agrícolas para el análisis de metales, consta de 5 etapas: Muestreo preliminar del área de estudio, muestreo definitivo, plan de muestreo, recolección de muestras y análisis de muestras.

\section{CONTRIBUCIÓN DE LOS AUTORESTodos}

los autores participaron en la redacción del manuscrito inicial, revisión bibliográfica, y en la revisión y aprobación del manuscrito final.

\section{CONFLICTO DE INTERESES}

Los autores declaran no tener conflicto de intereses.

\section{REFERENCIAS BIBLIOGRÁFICAS}

Burbano, H. 2016. "El suelo y su relación con los servicios ecosistémicos y la seguridad alimentaria”. Revista de Ciencias Agrícolas 33 (2): 117-124. DOI: 10.22267/rcia.163302.58.

Delince, W., R. Valdés, O. López, F. Guridi y M. I. Balbín. 2015. "Riesgo agroambiental por metales pesados en suelos con Cultivares de 
Oryza sativa L y Solanum tuberosum L". Revista Ciencias Técnicas Agropecuarias 24 (1): 44-50.

Díaz, S., R. Mayari, M. Espinoza y R. Hernández. 2005. "Metodología para el Muestreo y Manipulación de muestras de Aguas y Aguas Residuales en un Labmovil". Revistas CENIC. Ciencias Químicas 36: 1-5.

FAO (Organización de las Naciones Unidas para la Alimentación y la Agricultura). 2015. Los suelos sanos son la base para la producción de alimentos saludables. Organización de las Naciones Unidas para la Alimentación y la Agricultura. Informe. Roma (Italia).

Gómez, G. C. 2008. "Mundo: Lo que no se dice del arroz". Servindi 20 (1): 1-10.

Guzmán, A. R., O. Cruz y R. Valdés. 2019. “Efectos de la contaminación por metales pesados en un suelo con uso agrícola". Revista Ciencias Técnicas Agropecuarias 28(1): 1-9

Kelepertzis, E. 2014. “Accumulation of heavy metals in agricultural soils of Mediterranean: Insights from Argolida basin, Peloponnese, Greece". Geoderma 221-222: 82-90. DOI: 10.1016/j.geoderma.2014.01.007

MINAM (Ministerio del Ambiente). 2014. Guía para el Muestreo de Suelos. Guía. Lima (Perú).

MINAM (Ministerio del Ambiente). 2017. ¿Qué son los Estándares de Calidad Ambiental (ECA)? http://www.minam.gob.pe/estandares-decalidad-ambiental/que-son-los-estandaresde-calidad-ambiental-eca/ (Consultada el 23 de abril de 2021)

Peralta, J.R., M. L. Lopez, M. Narayan, G. Saupe y J. Gardea. 2009. "The biochemistry of environmental heavy metal uptake by plants: Implications for the food chain". The International Journal of Biochemistry \& Cell Biology 41: $1665-1677$. D O I : 10.1016/j.biocel.2009.03.005

Pérez, E. 2013. “Análisis de fertilidad de suelos en el laboratorio de Química del Recinto de Grecia,
Sede de Occidente, Universidad de Costa Rica”. Revista InterSedes 14 (29): 6-18.

Rueda, G., J. A. Rodríguez y R. Madriñán. 2011. “Metodologías para establecer valores de referencia de metales pesados en suelos agrícolas: Perspectivas para Colombia". Acta Agronómica 60 (3): 203-218.

Sharafati, F., M. Rafieian y R. Sharafati. 2016. “A review of heavy metals in rice (Oryza sativa) of Iran". Toxin Reviews 36(2): 147-153. DOI: $10.1080 / 15569543.2016 .1252932$ 